\title{
El desafío de escribir la sección de discusión de un artículo científico
}

\author{
Gastón Mougabure-Cueto
}

Laboratorio de Investigación en Triatominos, Centro de Referencia de Vectores, Ministerio de Salud de la Nación. Santa María de Punilla, Córdoba, Argentina. Consejo Nacional de Investigaciones Científicas y Técnicas (CONICET), Argentina.

Resumen. A diferencia de otras secciones más descriptivas, la sección Discusión de un artículo científico requiere un nivel de elaboración mayor. En esta sección se espera que se analicen los resultados en función de los objetivos y las hipótesis del estudio, se comparen estos resultados con los obtenidos en estudios previos y se establezcan los aportes del estudio al tema de investigación. Sin embargo, y a pesar de estos objetivos, los autores se enfrentan a diferentes problemas o incertidumbres tanto en el momento de escribir la discusión de un artículo propio como en el de orientar a los estudiantes cuando comienzan a escribir sus primeros manuscritos. La manera de vincular los resultados con las hipótesis, el orden en que se desarrollan los diferentes aspectos de la discusión y la posibilidad y grado de especulación científica son algunas de las incertidumbres que emergen al momento de abordar la sección. En este trabajo se presenta un método para desarrollar la sección Discusión de un artículo científico. La propuesta se basa en el análisis de hipótesis, en conceptos epistemológicos y en una división interna según diferentes niveles de análisis. Se propone un primer nivel de discusión interna y contrastación de hipótesis, un segundo nivel de discusión comparativa con el conocimiento existente del mismo orden y un tercer nivel en el que se discuten hipótesis en diferentes niveles de organización biológica. Estas propuestas pretenden guiar el desarrollo de una discusión y dar solución a los inconvenientes planteados al hacer explícita la contrastación de las hipótesis, ordenar la discusión e incentivar la especulación científica bajo criterios que limitan su exceso.

[Palabras clave: publicación científica, contrastación de hipótesis, explicación científica, explicación mecanística, predicción científica, especulación científica]

Aвstract. The challenge of writing the discussion section of a scientific article. Unlike other more descriptive sections, the discussion section of a scientific article requires a higher level of elaboration. This section is expected to discuss the results in terms of the objectives and hypotheses of the study, compare these results with those obtained in previous studies and establish the contributions of the study to the research topic. However, despite these objectives, the authors face different problems or uncertainties both when writing the discussion of their own article and in guiding students when they start to write their first manuscripts. The way in which the results are linked to the hypotheses, the order in which the different aspects of the discussion are developed and the possibility and degree of scientific speculation are some of the uncertainties that emerge when approaching the section. This paper presents a method for developing the discussion section of a scientific article. The proposal is based on the analysis of hypotheses, on epistemological concepts and on an internal division according to different levels of analysis. A first level of internal discussion and hypothesis testing, a second level of comparative discussion with existing knowledge of the same order, and a third level in which hypotheses are discussed at different levels of biological organization are proposed. These proposals are intended to guide the development of a discussion and gives solution to the inconveniences posed by making explicit the testing of hypotheses, ordering the discussion and encouraging scientific speculation under criteria that limit their excess.

[Keywords: scientific publication, hypothesis testing, scientific explanation, mechanistic explanation, scientific prediction, scientific speculation]

Editor asociado: Alejandro Farji-Brener 更 gmougabure@gmail.com
Recibido: 10 de Agosto de 2020

Aceptado: 2 de Octubre de 2020 


\section{INTRODUCCIÓN}

Escribir artículos científicos y publicarlos en revistas especializadas es una actividad central de los científicos, ya que allí comunican los resultados de sus investigaciones a la comunidad especializada. Junto con los resultados, los autores describen en detalle los materiales que utilizaron y la manera en que desarrollaron la investigación (i.e., diseño experimental, metodologías, experimentos, recolección y análisis de los datos) de manera que el estudio pueda ser repetido por otros investigadores. Si bien la difusión de los estudios y sus resultados es el objetivo principal de los manuscritos científicos, otros dos aspectos centrales de toda investigación son desarrollados en este tipo de artículos. Por un lado, se describen los marcos teóricoconceptuales, antecedentes, hipótesis y objetivos de la investigación desarrollada. Por otro lado, se discuten los resultados; esto generalmente incluye su interpretación, la comparación con trabajos previos y el aporte del estudio al tema general de investigación. Por lo general, todos estos aspectos de un artículo científico se desarrollan en secciones separadas, típicamente Introducción, Materiales y Métodos, Resultados y Discusión, aunque puede haber fusión de secciones y subsecciones.

Al momento de comenzar a escribir un artículo científico resulta necesario tener presente los requerimientos y alcances de cada sección. Las secciones Materiales y Métodos y Resultados son netamente descriptivas y en conjunto deben exponer de la manera más clara posible qué se hizo, para qué se hizo, cómo se hizo y qué se obtuvo; y, dentro de lo posible, no incorporar otra información. En estas secciones queda relativamente bien establecido cuál aspecto de la investigación se debe describir primero y cuál después (Branch and Villareal 2008; Harley et al. 2004). De esta manera, estas secciones no presentan mayores dificultades sobre lo que deben contener y, por lo tanto, sobre cómo escribirlas. Contrariamente, las secciones Introducción y Discusión están vinculadas con los aspectos teórico-conceptuales, interpretativos y lógicos del estudio, por lo que resulta menos claro qué información deben contener por lo que requieren de un nivel de elaboración mayor (Toft and Jaeger 1998).

En la sección Discusión se espera que se lleve a cabo una interpretación integral de los resultados en relación con los objetivos del estudio y con las hipótesis propuestas, una comparación con el conocimiento previo y que se establezca el aporte del estudio al marco teórico-conceptual de la línea de investigación (Lertzman 2002; Herley et al. 2004; FarjiBrener 2019a). Sin embargo, y a pesar de cierto consenso acerca de estos objetivos, los autores se enfrentan a diferentes problemas o incertidumbres al momento de abordar esta sección. El vínculo entre los resultados y las hipótesis parece resultar en particularmente problemático. La sección permite presentar uno de los procesos centrales en toda investigación científica, la contrastación de las hipótesis. Sin embargo, no siempre los autores establecen de manera explícita si cada hipótesis propuesta es corroborada o no, y por cuáles resultados del estudio (en este artículo se utiliza el término "corroboración" para indicar que una hipótesis ha resistido con éxito intentos de refutación y ha probado, hasta el momento, no ser falsa [Klimovsky 1994]). Esta ausencia resulta inconveniente para comunicar una investigación porque no le permite al lector determinar de forma cabal la relevancia del estudio respecto de las preguntas e hipótesis formuladas. Otro aspecto problemático es el orden en el que desarrollar los diferentes aspectos de la discusión, que no siempre resulta claro. El desorden atenta contra la lógica y claridad de la discusión como un conjunto, y dificulta la comunicación entre el autor y el lector. Por último, existe la incertidumbre acerca del grado permitido de especulación, entendida ésta como construir conjeturas o hipótesis más allá de los datos que se tienen al alcance (Bunge 1983). Luego de evaluar la asociación estrecha entre las hipótesis propuestas al comienzo de la investigación (las originales) y los datos producidos para contrastarlas, ¿es posible discutir hipótesis diferentes a las originales, es decir, menos relacionadas a los datos producidos?, y, si es posible, ¿hasta qué grado de independencia entre datos e hipótesis está permitido? Las posiciones de autores y revisores de manuscritos van desde discutir sólo las hipótesis estrechamente asociadas a los datos hasta permitir diferentes niveles de especulación. Sin embargo, para los que incentivan la especulación, el límite que determina el paso de la especulación aceptable a la no aceptable es confuso y la incertidumbre emerge cada vez que se aborda una discusión. Esto resulta inconveniente tanto porque hace difícil estructurar la discusión, como porque puede subestimar o sobreestimar los alcances teóricos del estudio. 
La epistemología es el estudio de las condiciones de producción y de validación del conocimiento científico (Klimovsky 1994). Este campo de estudio permitió establecer una variedad de métodos o tácticas empleadas para construir conocimiento científico, es decir, métodos científicos. Entre ellos, el método hipotético-deductivo establece un conjunto de procedimientos por los cuales en las ciencias fácticas se plantean los problemas y las hipótesis científicas y se ponen a prueba las hipótesis (Klimovsky 1994; Bunge 2005). Este método consiste en identificar el problema, proponer hipótesis, deducir sus consecuencias observacionales o predicciones, diseñar y ejecutar la prueba de la hipótesis, recolectar e interpretar los datos obtenidos, comparar los resultados de la prueba con las predicciones $\mathrm{y}$, en función de la comparación, abandonar o conservar la hipótesis (Klimovsky 1994; Bunge 2005). Si bien se trata de una estrategia general que guía la investigación científica, también se puede apelar a este método para optimizar la presentación escrita de una investigación. Así, por ejemplo, formular correctamente una hipótesis y hacer explícitas las predicciones y los objetivos de las pruebas permite comprender mejor un proyecto de investigación o los resultados de un estudio (Farji-Brener 2003, 2004).

El objetivo de este artículo es presentar un método para escribir o desarrollar la sección Discusión de un artículo científico. El método propuesto está basado en: 1) un análisis focalizado en las hipótesis, 2) nociones epistemológicas, lo que permitiría una mayor eficiencia lógica, explicativa y especulativa en comparación a otras formas de abordar la discusión, y 3) diferentes niveles de análisis respecto del problema estudiado. De esta manera, a diferencia de artículos previos que establecen qué aspectos se deberían tener en cuenta, y cuáles no, en la sección de discusión (Lertzman 1995; Toft and Jaeger 1998; Harley et al. 2004; Branch and Villarreal 2008; Farji-Brener 2019a), el presente artículo establece y describe una serie de etapas que estructuran y guían el desarrollo de la sección. Para una mayor compresión del método se presentará una investigación experimental simple y ficticia que se utilizará a lo largo del artículo para ejemplificar cada nivel de discusión propuesto. Dado que el método descripto en los siguientes párrafos sería aplicable sólo a estudios que ponen a prueba hipótesis o deductivos (Farji-Brener 2019b), el artículo también presenta una sección con sugerencias para adaptar este método a estudios descriptivos o que no ponen a prueba hipótesis (Farji-Brener 2019b).

\section{EL PRIMER PÁRRAFO}

Para comenzar la sección Discusión se suele utilizar el primer párrafo a modo de introducción (Lertzman 2002). Este párrafo se puede abordar desde perspectivas muy diferentes; una de ellas es presentar un resumen de lo estudiado y de los principales resultados ordenados según la lógica con la cual se concibió y llevó a cabo la investigación. Este tipo de párrafo inicial le aporta al lector claridad respecto de lo descripto en detalle en la sección Resultados, y, por otro lado, el material sobre el que se va a desarrollar la discusión propiamente dicha.

Luego del párrafo introductorio, propongo estructurar la sección de discusión en función de las hipótesis propuestas al comienzo del estudio y expuestas en la introducción del artículo. En primera instancia, resulta conveniente dividir conceptualmente la discusión en grandes partes, cada una correspondiendo a cada hipótesis. Luego, dentro de cada hipótesis, propongo una división en sub-secciones (no formalmente explicitadas) que corresponden a diferentes niveles de análisis tanto de los resultados como de las propias hipótesis.

\section{Primer nivel: Discusión INTERna Y CONTRASTACIÓN DE LAS HIPÓTESIS}

El primer nivel de discusión corresponde a interpretar los resultados obtenidos y a llevar a cabo el proceso de contrastación de hipótesis (Figura 1). Para esto, en primer lugar, se deben interpretar los resultados en el marco experimental y conceptual del estudio, y establecer si se ajustan, o no, a las predicciones deducidas de la hipótesis propuesta (Klimovsky 1994). En muchas investigaciones, una predicción es la hipótesis alternativa de la prueba estadística usada para evaluar los datos del estudio o experimento (Farji-Brener 2003). La idea del proceso de verificación de las predicciones es la de determinar si los resultados obtenidos son similares o no a los que se supusieron que se iban a obtener si la hipótesis biológica fuera verdadera. Luego, a partir del resultado de esta verificación se establecerá qué sucede con la hipótesis propuesta. Si lo predicho y lo obtenido coincidieran, entonces la hipótesis 


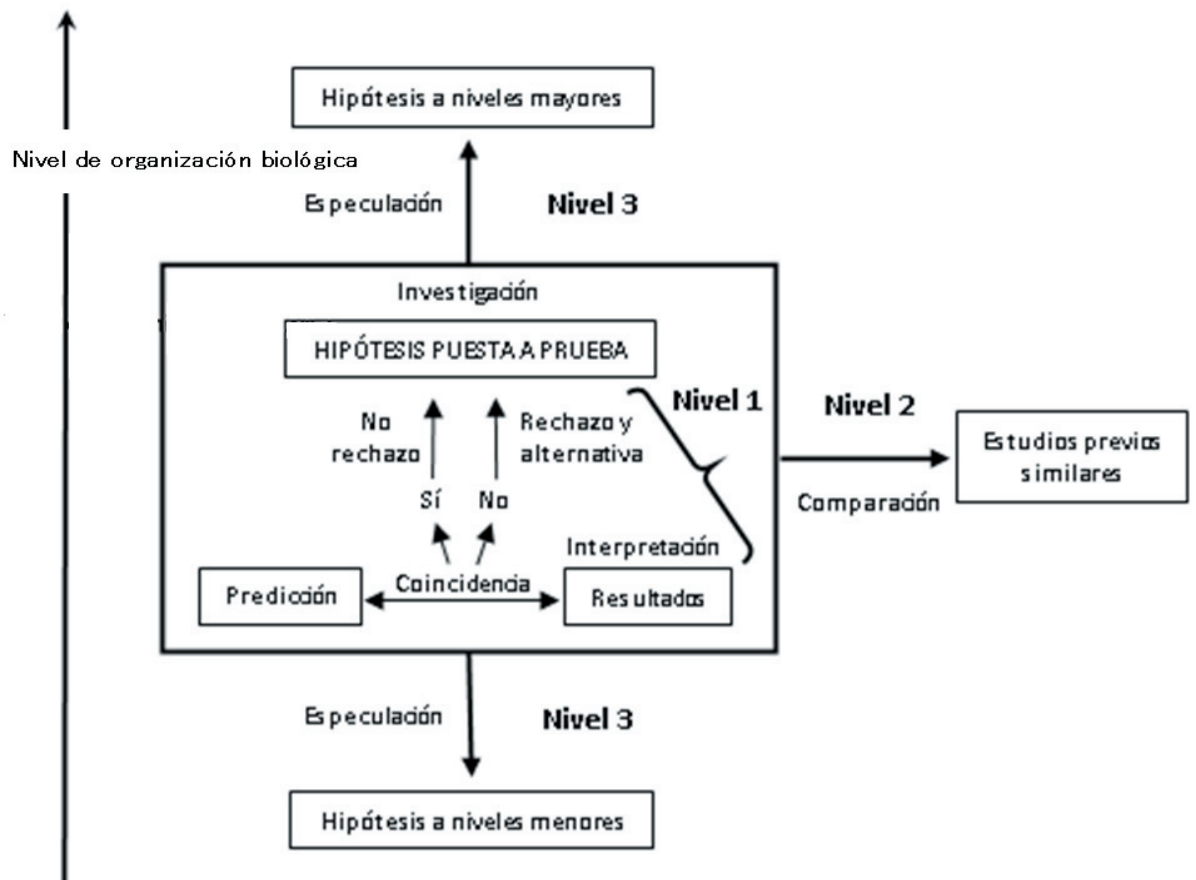

Figura 1. Esquema de los vínculos entre los tres niveles de discusión propuestos y la investigación realizada. Figure 1. Scheme of the links between the three levels of discussion proposed and the research carried out.

biológica no sería rechazada o refutada. Si no existiera tal coincidencia, la hipótesis sería rechazada y se recomienda proponer hipótesis biológicas alternativas. Este último ejercicio es clave, ya que lleva al investigador a proponer alternativas que no habría tenido en cuenta si los datos hubieran concordado con la predicción. Cabe destacar que la reflexión acerca de hipótesis alternativas es muy útil aun cuando haya coincidencia entre lo esperado y los obtenido, ya que permite exponer el abanico de explicaciones posibles de los resultados, además de la hipótesis original. En el marco de una versión más compleja del método hipotético-deductivo se podría no rechazar la hipótesis a pesar de la inconsistencia entre los resultados y las predicciones. En este caso, se deberían explicar las razones que permiten sostener la hipótesis propuesta (i.e., algún supuesto teórico no verificado, algún problema en la obtención de los datos, etc.) (Klimovsky 1994).

\section{Ejemplo}

En el contexto de una conocida asociación entre la especie de insectos dípteros $\mathrm{X}$ y el envés del follaje, se realizó un experimento para poner a prueba la hipótesis que postula "Los individuos de la especie $X$ se asocian al envés del follaje porque son fotofóbicos". La predicción evaluada fue "Los individuos de la especie X preferirán las zonas oscuras de un ambiente que en parte está iluminado y en parte a oscuras". El experimento consistió de un ensayo en el que un individuo de la especie $X$ camina durante 10 minutos sobre una superficie que tiene una mitad iluminada y la otra a oscuras, y se midió el tiempo que el individuo pasa en cada mitad. El ensayo control fue igual, pero con las dos mitades iluminadas. Se evaluaron 30 individuos en cada ensayo. Los resultados mostraron que el promedio del tiempo en la mitad oscura fue mayor que en la mitad iluminada, mientras que no hubo diferencias en el tiempo promedio entre las dos mitades del tratamiento control. Entonces, en la discusión se podría expresar que la preferencia por la mitad oscura y la falta de preferencia por alguna zona cuando la superficie estuvo homogéneamente iluminada indican que la orientación de los individuos estuvo influida por el nivel de iluminación, de manera que prefirieron la oscuridad. Estos resultados coinciden con la predicción. En consecuencia, se debería manifestar que los resultados recién discutidos corroboran la hipótesis propuesta.

Este proceso se debería repetir para cada consecuencia observacional, si se hubieran evaluado más de una predicción de la hipótesis. En este caso, si la consideración conjunta de los resultados asociados a cada 
predicción no permitiera tomar una decisión acerca de la contrastación de la hipótesis, por ejemplo, si algunos resultados rechazan y otros corroboran, sería interesante a) describir qué tipo de estudios futuros ayudarían a evaluar la hipótesis en cuestión y b) jerarquizar las predicciones según su capacidad de contrastación y establecer un juicio acerca de la veracidad de la hipótesis en función de las predicciones verificadas o refutadas.

\section{Segundo nivel: Discusión COMPARATIVA Y EL SOPORTE CIENTÍFICO DE LOS RESULTADOS E HIPÓTESIS}

El segundo nivel de discusión corresponde al análisis comparativo entre los resultados del estudio que se está presentando y aquellos de estudios previos que estudiaron, analizaron o discutieron fenómenos, preguntas o hipótesis similares (Figura 1). Esta comparación no debe perder el vínculo con la hipótesis original sobre la cual se está discutiendo (i.e., se debe evitar la simple enumeración de los resultados de trabajos similares). Para las hipótesis corroboradas, esta comparación está vinculada con las ampliaciones del soporte empírico y del soporte racional de la hipótesis; esto es, evaluar la coherencia entre los resultados e hipótesis en estudio y el conocimiento disponible del mismo orden (Bunge 2005). Sin embargo, este nivel es el que en menor medida se apelaría a herramientas epistemológicas para que guíen su desarrollo. En esta parte de la discusión conviene comenzar con los estudios previos más similares a la investigación presentada como, por ejemplo, el mismo fenómeno o proceso en el mismo grupo taxonómico. Luego, continuar con estudios cada vez más diferentes como, por ejemplo, el mismo proceso en diferentes grupos taxonómicos, procesos estrechamente vinculados al fenómeno estudiado, el mismo fenómeno en diferentes regiones geográficas o biomas, etc.

\section{Ejemplo}

Se podrían citar y comparar estudios sobre el comportamiento de orientación a la luz en otros insectos dípteros y luego, en otros tipos de insectos $\mathrm{u}$ otros tipos de organismos. De esta manera, se podría interpretar este comportamiento como una adecuación que podría ir más allá de la especie estudiada. También se podrían citar, comentar y comparar estudios sobre la ecología de la interacción de la especie $X$ con el follaje o sobre la orientación de la especie $X$ a otros estímulos, además del nivel de iluminación, que provienen del follaje. También se podrían considerar estudios en la especie $X$ o especies cercanas sobre la orientación respecto a otros estímulos visuales $\mathrm{y}$, tal vez, su vínculo con otros tipos de refugio. Las citas estarían agrupadas según el tipo de nexo con el estudio llevado a cabo (la orientación a la luz, la interacción díptero-follaje, la orientación a otros estímulos, la utilización de otro tipo de refugio, etc.). En todos los casos, es importante siempre relacionar esos estudios con la orientación a la luz de la especie $X$ y su vínculo con el follaje (i.e., con la hipótesis original propuesta) para que las referencias a otros trabajos no se conviertan en una simple lista de trabajos similares.

Si hay muchos estudios previos que permitan comparaciones, la elección de cuáles considerar se realizará según diferentes criterios. Entre estudios similares conviene discutir primero algunos más alejados en el tiempo, tal vez los trabajos pioneros, para luego focalizarse en los más cercanos en el tiempo. Considerando estudios que abordaron el mismo fenómeno desde diferentes perspectivas teóricas y metodológicas, conviene focalizarse en aquellos que utilizaron un abordaje similar al estudio que se está presentando; de los estudios que utilizaron otras herramientas experimentales, es recomendable citar solo aquellos que aportaron información relevante respecto de la pregunta formulada. Finalmente, si el fenómeno fue estudiado en diferentes grupos taxonómicos, el nivel de generalización que tendrá la discusión dependerá del interés particular que tiene la especie estudiada, del nivel de conocimiento que se tiene sobre el fenómeno estudiado, y en última instancia del interés de los propios investigadores en las especulaciones con elevado nivel de generalización biológica. Así, si el estudio se hubiera realizado sobre una especie con algún interés practico para los humanos como, por ejemplo, una especie plaga, entonces, la discusión se podría focalizar en la problemática vinculada con esa especie y ser más acotada respecto de la diversidad biológica que si el estudio se hubiera realizado sobre especies sin ese tipo de interés o especies modelo. Pero aun en los estudios sobre especies con algún interés práctico, algunos autores podrían tener la intención de discutir en función 
de la diversidad biológica, especular sobre rangos taxonómicos superiores y abordar generalizaciones biológicas de mayor orden. El alcance de la discusión también puede depender de la revista a la que se enviará el artículo. Si, por ejemplo, la revista es de ecología de insectos, entonces, la discusión se deberá acotar a los insectos. En revistas más conceptuales o generales se puede enfatizar los aportes generales al marco teórico que proporcionaron el estudio específico; por ejemplo, la evolución y el mantenimiento del comportamiento fotofóbico más allá de los insectos o la fotofobia en lo animales y su relación con el uso de refugios. En cualquier caso, si los autores quieren publicar sus resultados en una revista determinada, deberán adecuar la discusión a ella; por el contrario, si los autores desean abordar la discusión de una determinada manera, entonces, deberán elegir la revista acorde.

\section{Ejemplo}

Si la especie de díptero $X$ es vector de una enfermedad y la orientación a la luz ya fue estudiada en diferentes especies, se podría focalizar esta parte de la discusión en la relevancia de la reacción fotonegativa sobre la capacidad vectorial del insecto y en la epidemiologia de le enfermedad si el interés está en el rol de la especie X como vector. Con este mismo interés epidemiológico, también se podría focalizar en el efecto del desmonte sobre la disponibilidad de refugios y el desplazamiento de los vectores desde el ambiente silvestrehacia el ambienteurbano. Sin embargo, si el interés radica en las implicancias ecológicas de la reacción comportamental a la luz, esta parte de la discusión se podría centrar en la capacidad de los animales de utilizar la luz como información ambiental y su valor adaptativo (e.g., reducir mortalidad por desecación, eludir depredadores, etc.) y ampliar la discusión a otros organismos y discutir en términos de insectos, artrópodos, invertebrados, etc.

\section{TERCER NIVEL: El VÍNCUlO CON PROCESOS EN OTROS NIVELES DE ORGANIZACIÓN BIOLÓGICA}

El tercer nivel de discusión corresponde a la extensión del análisis a diferentes niveles de organización biológica partiendo desde los procesos e hipótesis evaluados en el estudio (Figura 1). Es la parte de la discusión en la que se habilita la especulación. Para esto, se propone apelar a la explicación y la predicción que pueden ocurrir como consecuencia de corroborar una hipótesis. En términos epistemológicos, la herramienta a utilizar es la relación entre explicación y predicción que se establece al utilizar un tipo de explicación científica (i.e., la explicación nomológico deductiva) y un tipo de método científico (i.e., el método hipotético-deductivo) (Klimovsky 1994; Marone and Bunge 1998). Según esta relación, una hipótesis corroborada permitiría predecir hechos. Esto ocurre cuando a partir de la hipótesis se deduce un hecho que aún no ha sido descripto. Este proceso deductivo se denomina predicción. A su vez, una hipótesis corroborada permitiría explicar hechos. En este caso, si un hecho ya conocido puede ser deducido a partir de la hipótesis recién corroborada, el hecho queda explicado por tal hipótesis. Este proceso deductivo se denomina explicación. Si este tipo de explicación se utiliza para vincular hechos e hipótesis ubicados en diferentes niveles de organización biológica, se podría obtener una explicación de tipo mecanística. Esto sucedería ya que a) los procesos y las hipótesis en niveles de organización menores podrían ser los mecanismos de los procesos e hipótesis en niveles de organización mayores, $\mathrm{y}$ b) los procesos e hipótesis en niveles mayores pueden ser deducidos de los procesos y las hipótesis en niveles menores. La ventaja de este proceder radica en que no sólo estaría utilizando las herramientas lógicas de un tipo explicación científica, sino también el poder de profundización en el conocimiento de la explicación por mecanismos (Marone and Bunge 1998).

Según lo recién planteado, se propone discutir hipótesis que trabajen a niveles de organización biológica menores respecto delos procesos e hipótesis evaluados y que permitan deducirlos, conformándose en sus posibles mecanismos (Figura 1). Así, si la hipótesis original tratara acerca del funcionamiento de un sistema biológico (e.g., una comunidad, un órgano, etc.), se podrían discutir hipótesis sobre los componentes de tal sistema (e.g., las especies, los tejidos, etc.). Estas hipótesis podrían haber sido corroboradas en trabajos previos con lo cual, lo hechos observados y las hipótesis corroboradas durante el estudio presentado quedarían explicadas desde el punto de vista mecanísmico. Sin embargo, si estas hipótesis a niveles menores aún no fueron propuestas, podrían presentarse por primera vez durante la discusión y proponerlas para su 
evaluación en trabajos futuros. Si la hipótesis original del estudio que se está presentando ya proponía mecanismos, se puede intentar profundizar la explicación mecanísmica siguiendo este proceder, disminuyendo aun más en los niveles de organización biológica.

\section{Ejemplo}

Se podría discutir la hipótesis que afirma que "La luz incrementa la actividad locomotora de la especie X de díptero". Esta hipótesis permite deducir la hipótesis original del estudio $\mathrm{y}$, a la vez, describe un proceso que, de ocurrir, determina un comportamiento fotofóbico, por lo que constituiría su mecanismo (i.e., el insecto pasa más tiempo en el ambiente oscuro que en el iluminado debido a que en la oscuridad camina más lento). Según cómo se desarrolle la redacción, la hipótesis también podría escribirse haciendo explícito el vínculo mecanísmico como "Los individuos de la especie $X$ de insectos dípteros muestran un comportamiento fotofóbico porque su actividad locomotora se incrementa con la luz". En cualquier caso, la discusión debería focalizarse sobre el conocimiento existente acerca del efecto de la luz sobre la actividad locomotora y su relación con la fotofobia. Podría haber otros mecanismos que determinen un comportamiento fotofóbico (e.g., evadir depredadores visuales) los cuales se podrían discutir o, por razones que exceden este artículo, los autores podrían elegir discutir solo el posible mecanismo descripto arriba.

Por otro lado, se propone discutir hipótesis que trabajen a niveles de organización mayores y que sean deducidas de las hipótesis corroboradas durante el estudio; de esta manera, las últimas conformarían los mecanismos de las primeras (Figura 1). Así, si la hipótesis original tuviera que ver con el funcionamiento de un sistema biológico (e.g., una comunidad, un órgano, etc.), se podrían discutir hipótesis sobre el sistema del cual forma parte (e.g., un ecosistema, un sistema de órganos, etc.). Si estas últimas hipótesis ya estuvieran propuestas por estudios previos, la hipótesis original conformaría su explicación mecanísmica. Si las hipótesis a niveles mayores aún no fueron propuestas, se podrían proponer por primera vez conformando una predicción.

\section{Ejemplo}

Se podría discutir la hipótesis que sostiene "Las hojas de mayor tamaño que generan más sombra favorecen el agrupamiento de la especie $X$ de díptero, incrementando su densidad poblacional en ambientes boscosos". Esta hipótesis es deducida de la hipótesis original del estudio $\mathrm{y}$, a la vez, describe un fenómeno que es determinado por el comportamiento fotofóbico, que se constituiría en su mecanismo. Dependiendo de cómo se desarrolle la redacción, la hipótesis también se podría escribir explicitando el vínculo mecanísmico como “Debido al comportamiento fotofóbico, la densidad poblacional de la especie $X$ se incrementa en regiones que posee vegetación con hojas de mayor tamaño y que generan mayor sombra". En cualquier caso, la discusión debería focalizarse sobre el conocimiento existente acerca de la asociación entre el tamaño de las hojas y la densidad poblacional de la especie $X$, y su relación con la fotofobia. En este caso no tendría mucho sentido discutir acerca de otros mecanismos, diferentes a la fotofobia, que expliquen la utilización de las hojas como refugios o la asociación entre tamaño de hoja y densidad poblacional porque excedería la presente discusión al perderse el vínculo con la hipótesis original.

Tanto en niveles de organización menores como mayores pueden ocurrir las siguientes situaciones al establecer la nueva hipótesis: a) podría haber sido propuesta en estudios previos para la especie estudiada, pero sin vincularla con el proceso que describe la hipótesis original (la fotofobia de la especie $X)$; entonces, en la discusión se establecería ese vínculo biológico, b) podría no haber sido propuesta para la especie estudiada, pero sí para otras especies; entonces, en la discusión se propondría la analogía/homología y se establecería el vínculo con la hipótesis original, c) podría haber sido propuesta y vinculada con el proceso de la hipótesis original en otras especies; entonces, en la discusión se propondría la analogía/homología con la especie estudiada, d) podría no haber sido propuestas nunca para ninguna especie; entonces, en la discusión se la presentaría por primera vez estableciendo claramente el vínculo biológico con el proceso descripto por la hipótesis original.

Este tercer nivel de discusión marca una posición clara acerca de la posibilidad de especulación en una discusión: el método propuesto incentiva la especulación científica (Figura 1). Sin embargo, y de mayor importancia que el posicionamiento dentro del debate, el método presentado establece 
límites de cierta precisión y objetividad a la especulación. Estos límites están determinados por los procesos de explicación y predicción. Básicamente, se permite discutir o proponer hipótesis que van más allá de los datos obtenidos, pero sólo las que estén vinculadas con las hipótesis originales corroboradas en la investigación mediante la lógica subyacente a los procesos de explicación y predicción. Bunge (1983) definió a la especulación como "formar hipótesis o conjeturas que van más allá de los datos a su alcance" y estableció los límites entre especulación sólida y descabellada. Una especulación es sólida si es compatible con el conocimiento científico previo y si puede ser confirmada o refutada por medio de una observación o un experimento. Aquella que no cumpla con estos dos requisitos, es descabellada. A diferencia de los criterios propuestos por Bunge, este manuscrito propone un criterio (el vínculo con las hipótesis originales del estudio mediante la explicación y la predicción) que establece cuán lejos de los datos deben estar las hipótesis para formar parte de la discusión.

Si la investigación hubiera puesto a prueba más de una hipótesis, se propone desarrollar los tres niveles de manera consecutiva para la primera hipótesis a discutir, luego desarrollar los tres niveles para la siguiente hipótesis y así hasta la última hipótesis. Este orden permite al lector seguir sin interrupción todas las implicancias de cada hipótesis evitando la dispersión en su atención.
Antes de presentar las conclusiones del trabajo se recomienda dedicar un párrafo a exponer y discutir las principales limitaciones del estudio llevado a cabo, esto es muy importante ya que determina los alcances de las conclusiones.

\section{EL ÚlTIMO PÁRRAFO: LAS CONCLUSIONES Y LA FINALIZACIÓN DE LA SECCIÓN DE DISCUSIÓN}

Para finalizar la sección Discusión a menudo se emplea el último párrafo con fines variados. Por ejemplo, para presentar las principales conclusiones del trabajo, los mayores aportes al campo de estudio y, eventualmente, las diferentes problemáticas que enmarcan al tema estudiado (e.g., sociales, económicas, etc.).

\section{Cómo ADAPTAR El MÉTOdo A ESTUDIOS DESCRIPTIVOS}

El método recién descripto se centra en estudios que ponen a prueba hipótesis. En función de ellas se analizan los resultados y se especula utilizando como herramientas el método hipotético-deductivo y el razonamiento deductivo. De esta manera, y siguiendo la clasificación de proyectos de investigación propuesta por Farji-Brener (2019b), el método sería aplicable sólo a estudios que contrastan hipótesis o deductivos; en los estudios que no contrastan hipótesis o descriptivos, la sección Discusión requiere otro

Tabla 1. Comparación del método propuesto para desarrollar la sección Discusión entre estudios deductivos y descriptivos.

Table 1. Comparison of the method proposed to develop the Discussion section between deductive and descriptive studies.

\begin{tabular}{lll}
\hline $\begin{array}{l}\text { Divisiones de la sección de } \\
\text { discusión }\end{array}$ & \multicolumn{1}{c}{ Estudio deductivo } & \multicolumn{1}{c}{ Estudio descriptivo $^{\text {a }}$} \\
\hline $\begin{array}{l}\text { Primer párrafo } \\
\text { Primer nivel de discusión }\end{array}$ & $\begin{array}{l}\text { Resumen ordenado de los resultados } \\
\text { Interpretación de los resultados en } \\
\text { el contexto del problema estudiado } \\
\text { y contrastación de las hipótesis } \\
\text { propuestas al comienzo del estudio }\end{array}$ & $\begin{array}{l}\text { Resumen ordenado de los resultados } \\
\text { Interpretación de los resultados en el } \\
\text { contexto del problema estudiado }\end{array}$ \\
Segundo nivel de discusión & $\begin{array}{l}\text { Análisis comparativo entre el estudio } \\
\text { presentado y los estudios previos }\end{array}$ & $\begin{array}{l}\text { Análisis comparativo entre el estudio } \\
\text { presentado y los estudios previos } \\
\text { Tercer nivel de discusión }\end{array}$ \\
$\begin{array}{l}\text { Propuesta de hipótesis a diferentes } \\
\text { niveles de organización biológica } \\
\text { respecto del fenómeno estudiado }\end{array}$ & $\begin{array}{l}\text { Propuesta de hipótesis que expliquen } \\
\text { los resultados del estudio }\end{array}$ \\
Párrafo final & $\begin{array}{l}\text { Principales conclusiones y aportes del } \\
\text { estudio }\end{array}$ & $\begin{array}{l}\text { Principales conclusiones y aportes del } \\
\text { estudio }\end{array}$ \\
\hline
\end{tabular}

${ }^{a}$ Clasificación según Farji-Brener (2019b) 
desarrollo. Sin embargo, es posible adaptar el método a partir de identificar las similitudes y las diferencias entre las discusiones de ambos tipos de estudio. El análisis de los resultados obtenidos y su comparación con estudios previos son aspectos comunes.

El papel de las hipótesis es un aspecto diferente. A diferencia de los estudios deductivos, las hipótesis que explican los resultados de las investigaciones descriptivas son planteadas luego del desarrollo del estudio para ser puestas a prueba en investigaciones posteriores y, por lo tanto, son presentadas en la sección Discusión (Farji-Brener 2019b). De esta manera, en los estudios descriptivos, la sección de discusión comienza con el primer párrafo, tal como fuera descripto anteriormente. A continuación se desarrolla el primer nivel, la discusión interna, en el que se interpretan los resultados en el contexto del problema biológico estudiado, pero sin la posibilidad de verificar predicciones y contrastar hipótesis (ya que éstas no fueron planteadas en la sección Introducción). Luego, se desarrolla el segundo nivel, la discusión comparativa, como ya fue descripto arriba pero solo focalizada en los resultados. Se continúa con el tercer nivel pero de una manera radicalmente diferente al descripto para estudios deductivos. En este nivel se presentan las hipótesis que intentan explicar los resultados del estudio llevado a cabo. A diferencia de las hipótesis del tercer nivel para un estudio deductivo, éstas no dependen de la lógica deductiva sino principalmente de la creatividad del investigador ayudado, por ejemplo, por analogías o la lógica inductiva (Bunge 1995). Finalmente, se desarrolla el último párrafo de la manera ya descripta. La Tabla 1 muestra un resumen comparativo del método propuesto para los dos tipos de estudios.

\section{¿Por qué es beneficioso el MÉTODO PROPUESTO?}

La sección Discusión de un manuscrito es de suma importancia en la presentación de una investigación. En dicha sección se debería interpretar los resultados obtenidos, cotejarlos con estudios previos y desarrollar diferentes análisis sobre los alcances y aportes de lo demostrado en el estudio. Yo propongo que la eficiencia en alcanzar tales objetivos aumenta si se emplean herramientas epistemológicas. En este contexto, los niveles de discusión propuestos permiten interpretar los objetivos recién descriptos en el marco del método científico en su versión hipotéticodeductiva como contrastación o verificación de las hipótesis propuestas, evaluación de la coherencia entre lo obtenido en el estudio y el conocimiento verificado y la proposición de nuevas hipótesis a diferentes niveles. Además de guiar el desarrollo de una discusión, esta perspectiva soluciona los inconvenientes planteados en la sección Introducción de este artículo, ya que hace explícita la contrastación de las hipótesis de la investigación, ordena la discusión eincentiva la especulación científica, a la vez que establece criterios que limitan su grado. Considero que esta propuesta permite una discusión exhaustiva y sólida de los resultados mediante herramientas que fortalecen la lógica, la explicación y la especulación.

Agradecimientos. Agradezco a P. A. Lobbia por las charlas que promovieron la concepción de este artículo y por la lectura crítica, los comentarios y los consejos que mejoraron versiones preliminares del manuscrito. Agradezco a los revisores anónimos cuyos comentarios y sugerencias mejoraron la calidad del manuscrito original.

\section{REFERENCIAS}

Branch, L. C., and D. Villarreal. 2008. Redacción de trabajos para publicaciones científicas. Ecología Austral 18:139150 .

Bunge, M. 1983. Speculation: Wild and sound. New Ideas in Psychology 1:3-6. https://doi.org/10.1016/0732118X(83)90024-7.

Bunge, M. 1995. La ciencia, su método y su filosofía. Quinta edición. De Bolsillo. Buenos Aires, Argentina.

Farji-Brener, A. G. 2003. Uso correcto, parcial e incorrecto de los términos" hipótesis" y" predicciones" en ecología. Ecología Austral 13:223-227.

Farji-Brener, A. G. 2004. ¿Son hipótesis las hipótesis estadísticas? Ecología Austral 14:201-203.

Farji-Brener, A. G. 2019a. Cómo escribir un manuscrito científico para ahuyentar lectores. Ecología Austral 29:094-098. https://doi.org/10.25260/EA.19.29.1.0.754.

Farji-Brener, A. G. 2019b. Una propuesta de marco conceptual para el desarrollo de proyectos de investigación en Entomología y ciencias afines. Revista Colombiana de Entomología 45:e7805 https://doi.org/10.25100/ socolen.v45i1.7805. 
Harley, C. D. G., M. A. Hixon, and L. A. Levin. 2004. Scientific writing and publishing-a guide for student. Bulletin of the Ecological Society of America 85:74-78. https://doi.org/10.1890/0012-9623(2004)85[74:SWAPGF]2.0.CO;2.

Klimovsky, G. 1994. Las desventuras del conocimiento científico: una introducción a la epistemología. Séptima edición. AZ Editora, Buenos Aires, Argentina.

Lertzman, K. 1995. Notes on writing papers and theses. Bulletin of the Ecological Society of America 76:86-90.

Marone, L., and M. Bunge. 1998. La explicación en ecología. Boletín de la Asociación Argentina de Ecología 7:35-37. Toft, C. A., and R. G. Jaeger. 1998. Writing for Scientific Journals I: The Manuscript. Herpetologica 54:S42-S54. 